\title{
The relation between ECG changes and troponin test in patients with non ST segment elevation acute coronary syndrome
}

\author{
Arwa M. Fuzi Al-Sarraf
}

From the Department of Medicine, College of Medicine, University of Mosul.

Correspondence: Arwa M. Fuzi Al-Sarraf. Lecturer, Department of Medicine, College of Medicine, University of Mosul, Mosul, Iraq. Email: drarwa1966@yahoo.com.

(Ann Coll Med Mosul 2013; 39 (1): 12-18).

Received: $29^{\text {th }}$ Feb. 2012; Accepted: 13 ${ }^{\text {th }}$ Jan. 2013.

\section{ABSTRACT}

Objectives: This article focuses on unstable angina and non ST segment elevation myocardial infarction. The objectives are to clarify different ECG pattern, to correlate it with troponin results, and to evaluate the effects of risk factors.

Methods: Seventy patients with clinical diagnosis of acute coronary syndrome were collected from coronary care unit in Ibn Sina Teaching Hospital in Mosul, 20 patients with ST segment elevation were excluded, and ECG changes were evaluated. Forty five patients had troponin I test, and the results were compared to their ECG changes. The risk factors and their numbers were studied. Data statistically analyzed using Z- 2 proportion and Chi-square tests.

Results: Fifty patients with a mean age 57 year (SD 13.2), including 26 males and 24 females, show (in order of frequency): ST depression (44\%), non-specific ECG (24\%), T wave inversion (22\%), and BBB $(10 \%)$. Fifteen $(33 \%)$ had positive troponin test, most of them were males $(80 \%)$, showed significant association with $T$ wave inversion ( $p$ value $=0.05$ ). Specific $E C G$ changes were associated with male gender, diabetes mellitus, hypertension, and old age ( $p$ value $<0.03$ ). Troponin positivity was associated with male sex ( $p$ value $=0.003$ ). Male gender and smoking were associated with positive troponin in significant ECG group ( $p$ value $<0.04$ ). All patients with positive troponin had at least two risk factors ( $p$ value $=0.002$ ).

Conclusion: ST segment depression was the most common ECG changes in non ST elevation acute coronary syndrome, but $\mathrm{T}$ wave inversion was more significantly associated with troponin positivity, which differs from previous studies. Male gender was the most critical risk factor; this was consistent with previous studies. Number of risk factor has important effect on ECG and troponin results.

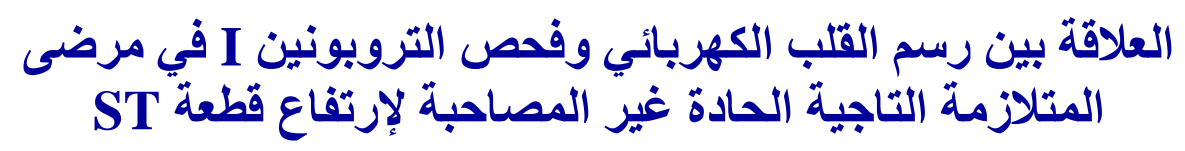

الخلاصة

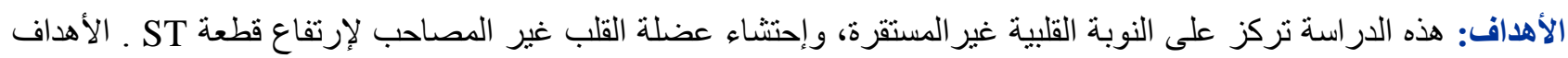

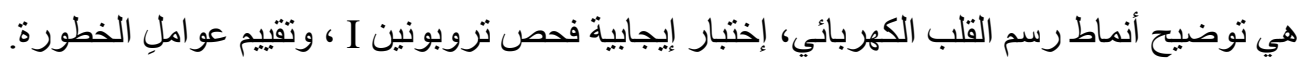

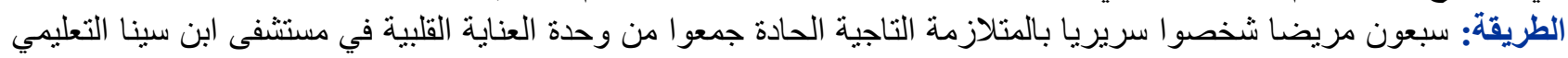

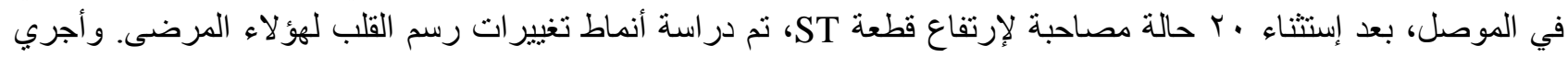

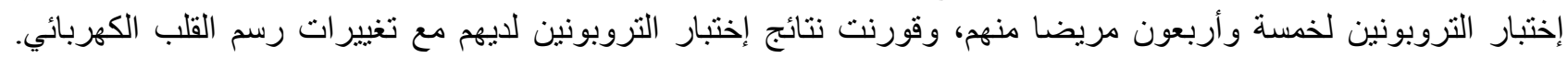

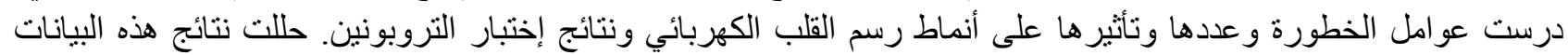

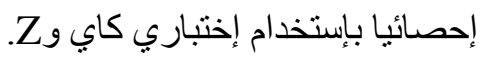

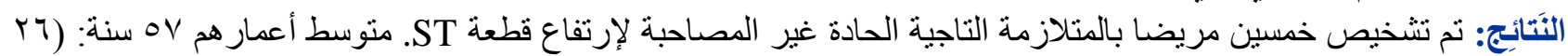

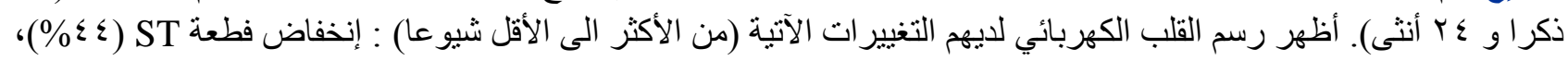

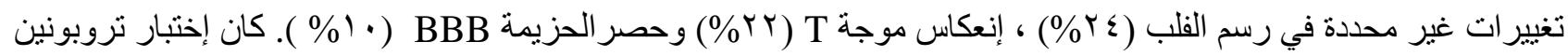

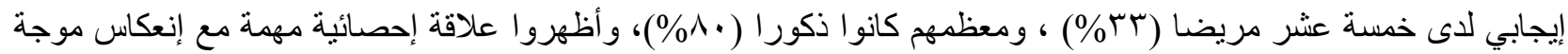




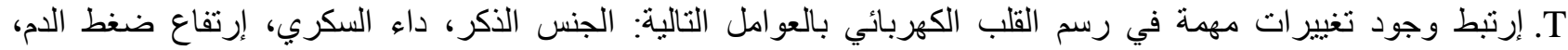

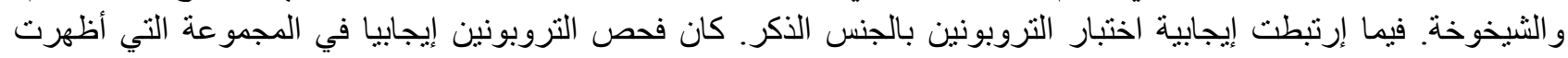

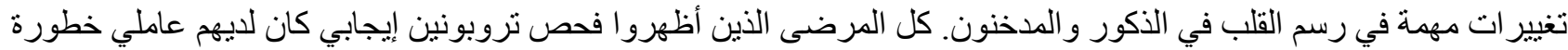
على الأقل.

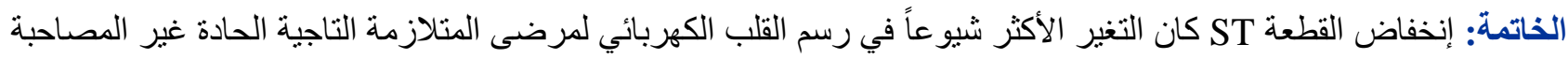

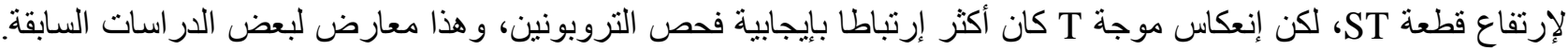

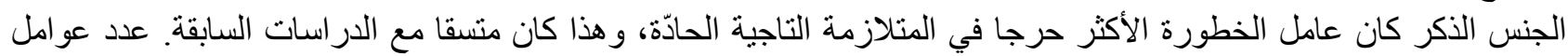

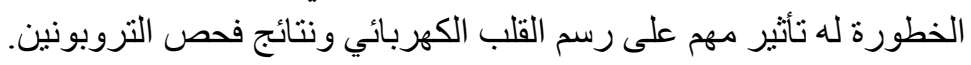

T he term acute coronary syndrome (ACS) refers to a range of acute myocardial ischemic states. It encompasses unstable angina (US), non ST segment elevation myocardial infarction (NSTEMI), and ST segment elevation infarction (STEMI) ${ }^{(1-5)}$.

The term ACS is useful because the initial presentation and early management is frequently similar ${ }^{(2)}$.

Unstable angina and NSTEMI are closely related conditions with clinical presentations that may be indistinguishable. Their distinction depends whether the ischemia is severe enough to cause myocardial damage and the release of detectable quantities of markers of myocyte necrosis. Cardiac troponin $I$ and $T$ are the preferred markers as they are more specific and reliable than creatine kinase or its isoenzyme creatine kinase $\mathrm{MB}^{(1)}$.

Troponin $(T, I, C)$ is found in striated and cardiac muscle. Because the cardiac and skeletal muscle isoforms of troponin $\mathrm{T}$ and I differ, they are known as "cardiac troponins". They are the preferred markers for the diagnosis of myocardial injury. They generally had similar sensitivity and specificity for the detection of myocardial injury. Unlike troponin I levels, troponin $\mathrm{T}$ levels may be elevated in patients with renal disease, polymyositis, and dermatomyositis (2,3,6-8). The cardiac troponins may remain elevated up to two weeks after symptoms onset which make them useful as late markers of recent acute myocardial infarction $^{(2,7)}$.

Acute coronary syndrome should be distinguished from stable angina, which develops during exertion and resolves at rest. In contrast US occur suddenly, often at rest or with minimal exertion, or at lesser degrees of exertion than the individual's previous angina (crescendo angina).
New onset angina is also considered US, since it suggests a new problem in a coronary artery ${ }^{(3)}$.

The cardinal symptom of decreased blood flow to the heart is chest pain experienced as tightness around the chest and radiating to the left arm and left angle of the jaw ${ }^{(3)}$.

In the setting of acute chest pain the electrocardiogram (ECG) is the investigation that most reliably distinguishes between various causes $^{(3,9)}$. If this indicate acute heart damage (elevation in ST segment or new left bundle branch block), angioplasty or thrombolysis is indicated immediately. In the absence of such changes, it is not possible to distinguish between US and NSTEMI ${ }^{(3)}$. If the ECG does not show typical changes, the term "non ST segment elevation ACS" (NSTE-ACS) is applied ${ }^{(3)}$. They may rather have persistent or transient ST segment depression or $\mathrm{T}$ wave inversion, flat $\mathrm{T}$ wave, pseudo-normalization of $T$ wave, or non-specific ECG changes. They may also have a normal ECG at presentation. ST segment shift and $T$ wave changes are the most reliable ECG indicators of unstable coronary disease ${ }^{(10,11)}$. Transient episodes of bundle branch block (BBB) occasionally occur during ischemic attack.

Different ways have been used to stratify risk in ACS patients. Simple risk stratification has been proposed in, according to the presence or absence of some key prognostic factors. These include older age, markers of severe ischemia (positive troponin, dynamic ST segment changes, ischemic recurrences on adequate treatment), signs of hemodynamic and electrical instability, signs of myocardial dysfunction, and co morbidities like diabetes mellitus $(\mathrm{DM})^{(12)}$.

The study objectives are to record different ECG changes in patients with NSTE- ACS, to study the positivity of serum troponin I in them, and to find 
the relation between these data. The effects of risk factors on the ECG changes and troponin positivity will be evaluated.

\section{PATIENTS AND METHODS}

Seventy patients with acute ischemic chest pain were collected from CCU in Ibn Sina teaching hospital in Mosul. Inclusion criteria included: recent destabilization of pervious stable angina, anginal pain at rest, and new onset angina. At time of admission ECG was conducted to all of these patients, those with ST elevation or $Q$ wave were excluded from the study.

Fifty patients were diagnosed as acute coronary syndrome without ST elevation (NSTE-ACS); after taking their consent, ECG records were carefully analyzed and classified into four groups:

- Normal or non-specific ECG changes.

- ECG with significant ST depression i.e. ST segment depression $>1 \mathrm{~mm}$ in two or more contiguous leads, ( $\mathrm{J}$ point horizontal or down sloping depression considering PT as isoelectrical line) with or without associated $T$ wave inversion ${ }^{(10)}$.

- Inverted $\mathrm{T}$ waves $>1 \mathrm{~mm}$ in leads with predominant $\mathrm{R}$ waves and deep symmetrical inversion of the $T$ waves in the anterior chest leads.

- New BBB.

At least 6 hours following the attack of chest pain, forty five patients with non ST elevation ACS (NSTE-ACS) had serum trponin I measurement in Ibn Sina laboratory. Results were considered either as troponin positive $(>0.01 \mathrm{mg} / \mathrm{dl})$ or troponin negative $(<0.01 \mathrm{mg} / \mathrm{dl})$.

Detailed history was taken with emphasis on diabetes mellitus, hypertension, current smoking, and known hyperlipidrmia. Also previous history of coronary or cerebrovascular disease, and positive family history of vascular disease. Although fasting blood sugar and lipid profile was tested at time of admission but it was not included in the study because of the possible effects of the new ischemia on their results.

The relation between ECG changes and tropnin positivity was studied, and compared in both male and female.

The effects of risk factors including male gender, DM, hypertension, current smoking, dyslipidemia, age above 60 years, and previous personal or family history of vascular diseases on both ECG changes and troponin positivity were studied.

The number of risk factors in NSTE-ACS patients was compared in both ECG groups and troponin results.

\section{Statistics}

Z-2 proportion test was applied to study the $p$ value and $95 \%$ confidence interval was calculated for all values. Chi square test also used to study the effect of number of risk factors on ECG changes and troponin positivity.

For both tests $P$ value equal to or less than 0.05 is considered significant.

\section{RESULTS}

Fifty patients with ACS without ST elevation were collected, including 26 male and 24 female, aged between 30-92 years (SD 13.2), with mean age 57 year old. Twelve patients (24\%) showed nonspecific changes, the other 38 patients showed in order of frequency: ST depression $44 \%, \mathrm{~T}$ inversion 22\% and BBB 10\%.

The 45 patients with NSTE-ACS had troponin test, 15 (33\%) showed positive results and considered as NSTEMI, 30 (67\%) patients were troponin negative and considered as unstable angina. $80 \%$ of troponin positive patients were males, while $60 \%$ of troponine negative patients were females ( $p$ value $=0.01)$, (Table 1).

No special ECG pattern was associated with troponin positivity in general, except for male patients who show significant association between $\mathrm{T}$ inversion and troponin positivity ( $p$ value $=0.05$ ), (Table 2).

Table 1. ECG pattern in patients with NSTE-ACS.

\begin{tabular}{|c|c|c|}
\hline \multirow{2}{*}{ ECG changes } & \multicolumn{2}{|c|}{$\begin{array}{c}\text { No. of } \\
\text { patients } 50(\%)\end{array}$} \\
\hline & $\begin{array}{c}M \\
26(52 \%)\end{array}$ & $\begin{array}{c}F \\
24(48 \%)\end{array}$ \\
\hline \multirow{2}{*}{ Non-specific changes } & \multicolumn{2}{|c|}{$\begin{array}{c}12 \\
24 \%\end{array}$} \\
\hline & $\begin{array}{c}6 \\
5 \% \\
\end{array}$ & $\begin{array}{c}6 \\
50 \% \\
\end{array}$ \\
\hline \multirow{2}{*}{ ST depression } & \multicolumn{2}{|c|}{$\begin{array}{c}1 \\
22 \\
44 \%\end{array}$} \\
\hline & $\begin{array}{c}11 \\
50 \% \\
\end{array}$ & $\begin{array}{c}11 \\
50 \% \\
\end{array}$ \\
\hline \multirow{2}{*}{$\mathrm{T}$ inversion } & \multicolumn{2}{|c|}{$\begin{array}{c}11 \\
22 \% \\
\end{array}$} \\
\hline & $\begin{array}{c}6 \\
54 \% \\
\end{array}$ & $\begin{array}{c}5 \\
46 \% \\
\end{array}$ \\
\hline \multirow{2}{*}{ New BBB } & \multicolumn{2}{|c|}{$\begin{array}{c}5 \\
10 \% \\
\end{array}$} \\
\hline & $\begin{array}{c}3 \\
54 \%\end{array}$ & $\begin{array}{c}2 \\
46 \% \\
\end{array}$ \\
\hline
\end{tabular}


The following risk factors were detected in patients with ACS, in order of frequency: hypertension $(51 \%)$, previous history of vascular disease $(42 \%)$, DM $(40 \%)$, age 60 years $(31 \%)$, current smoking and hyperlipidemia (11\%) and family history (9\%). Only (4\%) of them had no risk factors, (Table 3).

Table 2. Relation between ECG changes and troponin result.

\begin{tabular}{|c|c|c|c|c|c|c|c|c|}
\hline \multirow{2}{*}{ ECG changes } & \multicolumn{2}{|c|}{$\begin{array}{l}\text { No. of patients } \\
45(100 \%)\end{array}$} & \multicolumn{2}{|c|}{$\begin{array}{c}\text { Troponin positive } \\
15(33 \%)\end{array}$} & \multicolumn{2}{|c|}{$\begin{array}{c}\text { Troponin negative } \\
30(67 \%)\end{array}$} & \multirow{2}{*}{\multicolumn{2}{|c|}{$\begin{array}{c}\text { P value } \\
0.01^{\star *}\end{array}$}} \\
\hline & $\begin{array}{c}M \\
24(53 \%)\end{array}$ & $\begin{array}{c}F \\
21(47 \%)\end{array}$ & $\begin{array}{c}M \\
12^{\star \star}(80 \%)\end{array}$ & $\begin{array}{c}F \\
3(20 \%)\end{array}$ & $\begin{array}{c}M \\
12(40 \%)\end{array}$ & $\begin{array}{c}F \\
18(60 \%)\end{array}$ & & \\
\hline \multirow{2}{*}{ Non-specific changes } & \multicolumn{2}{|c|}{$\begin{array}{c}12 \\
27 \%\end{array}$} & \multicolumn{2}{|c|}{$\begin{array}{c}4 \\
27 \% \\
\end{array}$} & \multicolumn{2}{|c|}{$\begin{array}{c}8 \\
27 \% \\
\end{array}$} & \multicolumn{2}{|c|}{1} \\
\hline & $\begin{array}{c}6 \\
50 \% \\
\end{array}$ & $\begin{array}{c}6 \\
50 \% \\
\end{array}$ & $\begin{array}{c}3 \\
75 \% \\
\end{array}$ & $\begin{array}{c}1 \\
25 \% \\
\end{array}$ & $\begin{array}{c}3 \\
37 \% \\
\end{array}$ & $\begin{array}{c}5 \\
63 \% \\
\end{array}$ & 0.3 & 0.28 \\
\hline \multirow{2}{*}{ ST depression } & \multicolumn{2}{|c|}{$\begin{array}{c}19 \\
42 \% \\
\end{array}$} & \multicolumn{2}{|c|}{$\begin{array}{c}4 \\
27 \% \\
27 \%\end{array}$} & \multicolumn{2}{|c|}{$\begin{array}{c}15 \\
50 \% \\
\end{array}$} & \multicolumn{2}{|c|}{0.11} \\
\hline & $\begin{array}{c}10 \\
53 \% \\
\end{array}$ & $\begin{array}{c}9 \\
47 \% \\
\end{array}$ & $\begin{array}{c}3 \\
75 \% \\
\end{array}$ & $\begin{array}{c}1 \\
25 \% \\
\end{array}$ & $\begin{array}{c}7 \\
47 \% \\
\end{array}$ & $\begin{array}{c}8 \\
53 \% \\
\end{array}$ & 0.7 & 0.053 \\
\hline \multirow{2}{*}{$\mathrm{T}$ inversion } & \multicolumn{2}{|c|}{$\begin{array}{c}10 \\
22 \% \\
\end{array}$} & \multicolumn{2}{|c|}{$\begin{array}{c}5 \\
33 \% \\
\end{array}$} & \multicolumn{2}{|c|}{$\begin{array}{c}5 \\
17 \% \\
\end{array}$} & \multicolumn{2}{|c|}{0.2} \\
\hline & $\begin{array}{c}5 \\
50 \% \\
\end{array}$ & $\begin{array}{c}5 \\
50 \% \\
\end{array}$ & $\begin{array}{c}4^{*} \\
80 \% \\
\end{array}$ & $\begin{array}{c}1 \\
20 \% \\
\end{array}$ & $\begin{array}{c}1 \\
20 \% \\
\end{array}$ & $\begin{array}{c}4 \\
80 \% \\
\end{array}$ & $0.050^{*}$ & 0.4 \\
\hline \multirow{2}{*}{ New BBB } & \multicolumn{2}{|c|}{$\begin{array}{c}4 \\
9 \% \\
9\end{array}$} & \multicolumn{2}{|c|}{$\begin{array}{c}2 \\
13 \% \\
\end{array}$} & \multicolumn{2}{|c|}{$\begin{array}{c}2 \\
7 \% \\
\end{array}$} & \multicolumn{2}{|c|}{0.5} \\
\hline & $\begin{array}{c}3 \\
75 \% \\
\end{array}$ & $\begin{array}{c}1 \\
25 \% \\
\end{array}$ & $\begin{array}{c}2 \\
100 \% \\
\end{array}$ & 0 & $\begin{array}{c}1 \\
50 \% \\
\end{array}$ & $\begin{array}{c}1 \\
50 \% \\
\end{array}$ & 0.2 & 0.3 \\
\hline
\end{tabular}

${ }^{*}$ Chi square test. Significant $p=<0.05 .{ }^{*} Z-2$ proportion test. Significant $p=<0.05 .95 \%$ confidence interval $0.05 \%-46 \%$.

Table 3. Effects of risk factors on ECG patterns and troponin positivity.

\begin{tabular}{|c|c|c|c|c|c|c|c|c|c|}
\hline \multirow{2}{*}{$\begin{array}{l}\text { Risk } \\
\text { factors }\end{array}$} & \multicolumn{3}{|c|}{$\begin{array}{l}\text { No. of patients } \\
45(100 \%)\end{array}$} & \multicolumn{2}{|c|}{$\begin{array}{c}\text { Troponin positive } \\
15(33 \%)\end{array}$} & \multicolumn{2}{|c|}{$\begin{array}{l}\text { Troponin negative } \\
30(67 \%)\end{array}$} & \multicolumn{2}{|c|}{$\begin{array}{c}\text { P value }=0.001 \\
\text { CI } 95 \%=(-52 \%)-(-13 \%)\end{array}$} \\
\hline & $\begin{array}{l}\text { SIG ECG } \\
33(73 \%)\end{array}$ & $\begin{array}{l}\text { NS ECG } \\
12(27 \%)\end{array}$ & $\begin{array}{l}\text { P value } \\
\mathrm{Cl} \mathrm{95 \%}\end{array}$ & $\begin{array}{l}\text { SIG ECG } \\
11(24 \%)\end{array}$ & $\begin{array}{c}\text { NS ECG } \\
4(9 \%)\end{array}$ & $\begin{array}{l}\text { SIG ECG } \\
22(49 \%)\end{array}$ & $\begin{array}{l}\text { NS ECG } \\
8(18 \%)\end{array}$ & 1 & 1 \\
\hline \multirow{2}{*}{ Male } & \multicolumn{3}{|c|}{$\begin{array}{c}24 \\
53 \%\end{array}$} & \multicolumn{2}{|c|}{$\begin{array}{l}12^{*} \\
80 \%\end{array}$} & \multicolumn{2}{|c|}{$\begin{array}{c}12 \\
40 \%\end{array}$} & \multicolumn{2}{|c|}{$\begin{array}{c}1 \\
0.003^{*} \\
13 \%-66 \%\end{array}$} \\
\hline & $\begin{array}{c}18^{*} \\
40 \%\end{array}$ & $\begin{array}{c}6 \\
13 \% \\
\end{array}$ & $\begin{array}{l}0.003 \\
9-44 \%\end{array}$ & $\begin{array}{c}9^{*} \\
82 \%\end{array}$ & $\begin{array}{c}3 \\
75 \%\end{array}$ & $\begin{array}{c}9 \\
41 \%\end{array}$ & $\begin{array}{c}3 \\
38 \%\end{array}$ & $\begin{array}{c}0.009 \\
10-45 \%\end{array}$ & 0.1 \\
\hline \multirow{2}{*}{ DM } & \multicolumn{3}{|c|}{$\begin{array}{c}18 \\
40 \%\end{array}$} & \multicolumn{2}{|c|}{$\begin{array}{c}6 \\
40 \%\end{array}$} & \multicolumn{2}{|c|}{$\begin{array}{c}1 \\
12 \\
40 \%\end{array}$} & \multicolumn{2}{|c|}{1} \\
\hline & $\begin{array}{c}13 \\
29 \%\end{array}$ & $\begin{array}{c}5 \\
11 \%\end{array}$ & $\begin{array}{c}0.03 \\
1-33 \%\end{array}$ & $\begin{array}{c}4 \\
36 \%\end{array}$ & $\begin{array}{c}2 \\
50 \%\end{array}$ & $\begin{array}{c}9 \\
41 \%\end{array}$ & $\begin{array}{c}3 \\
38 \%\end{array}$ & 0.7 & 0.6 \\
\hline \multirow{2}{*}{ HT } & \multicolumn{3}{|c|}{$\begin{array}{c}33 \\
51 \%\end{array}$} & \multicolumn{2}{|c|}{$\begin{array}{c}1 \\
7 \\
47 \%\end{array}$} & \multicolumn{2}{|c|}{$\begin{array}{c}1 \\
16 \\
53 \%\end{array}$} & \multicolumn{2}{|c|}{0.6} \\
\hline & $\begin{array}{c}17^{*} \\
38 \%\end{array}$ & $\begin{array}{c}6 \\
13 \%\end{array}$ & $\begin{array}{l}0.006 \\
7-41 \%\end{array}$ & $\begin{array}{c}5^{*} \\
45 \%\end{array}$ & $\begin{array}{c}2 \\
50 \%\end{array}$ & $\begin{array}{c}12 \\
55 \%\end{array}$ & $\begin{array}{c}4 \\
50 \%\end{array}$ & 0.6 & 1 \\
\hline \multirow{2}{*}{ Smoking } & \multicolumn{3}{|c|}{$\begin{array}{c}5 \\
11 \%\end{array}$} & \multicolumn{2}{|c|}{$\begin{array}{c}3 \\
20 \%\end{array}$} & \multicolumn{2}{|c|}{$\begin{array}{c}1 \\
2 \\
7 \%\end{array}$} & \multicolumn{2}{|c|}{0.2} \\
\hline & $\begin{array}{c}3 \\
7 \%\end{array}$ & $\begin{array}{c}2 \\
4 \%\end{array}$ & 0.6 & $\begin{array}{c}3 \\
27 \%\end{array}$ & 0 & 0 & $\begin{array}{c}2 \\
25 \%\end{array}$ & $\begin{array}{c}0.04^{\star} \\
0.1-22 \%\end{array}$ & 0.1 \\
\hline \multirow{2}{*}{ Age $>60$} & \multicolumn{3}{|c|}{$\begin{array}{c}14 \\
31 \%\end{array}$} & \multicolumn{2}{|c|}{$\begin{array}{c}6 \\
40 \%\end{array}$} & \multicolumn{2}{|c|}{$\begin{array}{c}1 \\
27 \%\end{array}$} & \multicolumn{2}{|c|}{0.3} \\
\hline & $\begin{array}{c}11^{*} \\
24 \%\end{array}$ & $\begin{array}{c}3 \\
7 \%\end{array}$ & $\begin{array}{c}0.16 \\
3-32 \%\end{array}$ & $\begin{array}{c}5^{*} \\
45 \%\end{array}$ & $\begin{array}{c}1 \\
25 \%\end{array}$ & $\begin{array}{c}6 \\
27 \%\end{array}$ & $\begin{array}{c}2 \\
25 \%\end{array}$ & 0.3 & 1 \\
\hline \multirow{2}{*}{$\begin{array}{l}\text { Family } \\
\text { history }\end{array}$} & \multicolumn{3}{|c|}{$\begin{array}{c}4 \\
9 \%\end{array}$} & \multicolumn{2}{|c|}{$\begin{array}{c}2 \\
13 \% \\
\end{array}$} & \multicolumn{2}{|c|}{$\begin{array}{c}1 \\
2 \\
7 \%\end{array}$} & & \\
\hline & $\begin{array}{c}2 \\
4.5 \% \\
\end{array}$ & $\begin{array}{c}2 \\
4.5 \% \\
\end{array}$ & --- & $\begin{array}{c}2 \\
18 \%\end{array}$ & 0 & 0 & $\begin{array}{c}2 \\
25 \%\end{array}$ & 0.1 & 0.1 \\
\hline Previous & & $\begin{array}{c}19 \\
42 \%\end{array}$ & & & & & & & \\
\hline $\begin{array}{l}\text { vascular } \\
\text { diseases }\end{array}$ & $\begin{array}{c}14 \\
31 \%\end{array}$ & $\begin{array}{c}5 \\
11 \%\end{array}$ & $\begin{array}{c}0.16 \\
3-36 \%\end{array}$ & $\begin{array}{c}4 \\
45 \%\end{array}$ & $\begin{array}{c}2 \\
50 \%\end{array}$ & $\begin{array}{c}10 \\
45 \%\end{array}$ & $\begin{array}{c}3 \\
38 \%\end{array}$ & 0.6 & 0.6 \\
\hline linid & & $\begin{array}{c}5 \\
11 \%\end{array}$ & & & & & & & \\
\hline Lipıds & $\begin{array}{c}3 \\
7 \%\end{array}$ & $\begin{array}{c}2 \\
4 \%\end{array}$ & 0.6 & $\begin{array}{c}1 \\
2 \%\end{array}$ & 0 & $\begin{array}{c}2 \\
9 \%\end{array}$ & $\begin{array}{c}2 \\
25 \%\end{array}$ & 1 & 1.0 \\
\hline & & $\begin{array}{c}2 \\
4 \% \\
\end{array}$ & & & & & & & \\
\hline No risk & $\begin{array}{c}2 \\
4 \%\end{array}$ & 0 & 0.1 & 0 & 0 & $\begin{array}{c}2 \\
9 \%\end{array}$ & 0 & & \\
\hline
\end{tabular}

$\mathrm{SIG}=$ significant, $\mathrm{NS}=$ non-specific, $\mathrm{DM}=$ diabetes mellitus, $\mathrm{HT}=$ hypertension. 
There was significant association between specific ECG changes and male sex ( $p$ value $=$ 0.003), diabetes mellitus $(p$ value $=0.03)$, hypertension $(p$ value $=0.006)$, and older age $(p$ value $=0.01$ )

NSTEMI (trponin positivity) was more common in males $80 \%$ ( $p$ value $=0.027$ ).

Those with NSTEMI who showed specific ECG changes were more likely to be male ( $p$ value $=$ 0.009 ) and smokers ( $p$ value $=0.04$ )
Patients with non-specific ECG changes who have positive troponin test, had the following risk factors: $75 \%$ were males, $50 \%$ had $\mathrm{DM}, \mathrm{HT}$, or previous history of vascular disease.

All patients with NSTEMI (troponin positive) had at least two risk factors, while $70 \%$ of unstable angina (troponin negative) had 2 or more risk factors ( $p$ value $=0.002)$, (Table 4$)$.

Table 4. Effects of number of risk factors on ECG patterns and troponin results.

\begin{tabular}{|c|c|c|c|c|c|}
\hline & \multicolumn{2}{|c|}{$\begin{array}{c}\text { Troponin positive } \\
15\end{array}$} & \multicolumn{2}{|c|}{$\begin{array}{c}\text { Troponin negative } \\
30\end{array}$} & \multirow{2}{*}{$\begin{array}{l}\text { Total } \\
45\end{array}$} \\
\hline & $\begin{array}{c}\text { Significant ECG } \\
11\end{array}$ & $\begin{array}{c}\text { Non-specific ECG } \\
4\end{array}$ & $\begin{array}{c}\text { Significant ECG } \\
22\end{array}$ & $\begin{array}{c}\text { Non-specific ECG } \\
8 \\
\end{array}$ & \\
\hline \multirow{2}{*}{$\geq 2 \mathrm{RF}$} & \multicolumn{2}{|c|}{$\begin{array}{c}15 \\
100 \% \\
\end{array}$} & \multicolumn{2}{|c|}{$\begin{array}{l}21 \\
70 \% \\
\end{array}$} & \multirow{2}{*}{36} \\
\hline & $\begin{array}{c}11 \\
100 \%\end{array}$ & $\begin{array}{c}4 \\
100 \% \\
\end{array}$ & $\begin{array}{c}14 \\
63 \% \\
\end{array}$ & $\begin{array}{c}7 \\
87 \% \\
\end{array}$ & \\
\hline \multirow{2}{*}{$<2 R F$} & \multicolumn{2}{|c|}{0} & \multicolumn{2}{|c|}{$\begin{array}{c}9 \\
30 \% \\
\end{array}$} & \multirow{2}{*}{9} \\
\hline & 0 & 0 & $\begin{array}{c}7 \\
32 \%\end{array}$ & $\begin{array}{c}2 \\
25 \%\end{array}$ & \\
\hline $\mathrm{P}$ value* & \multicolumn{5}{|c|}{0.002} \\
\hline
\end{tabular}

\section{DISCUSSION}

The NSTE-ACS which is a life threatening disorder is a major cause of emergency medical care admissions in USA ${ }^{(7,13)}$, nearly $60 \%$ of these admissions are among persons greater than 65 years old, and almost half occur in women ${ }^{(7)}$. In our study $14(31 \%)$ patients with NSTE-ACS were more than 60 year of age, the number of male and female patients was nearly equal.

There was no difference between male and female in their ECG pattern. ST segment depression was the most common ECG changes in this syndrome, followed by non-specific changes, $T$ wave inversion, and lastly BBB. The predictive value of the ECG varies markedly, depending on the baseline risk (pretest probability) for coronary artery disease in a given patient. The number and magnitude of ECG abnormalities also affect sensitivity and specificity ${ }^{(2)}$.

The magnitude of an ECG abnormality affects diagnostic accuracy. One group of investigators (14), found that the diagnosis of NSTEMI is more likely in patients with chest pain who's ECG showed ST segment depression in three or more leads or ST segment depression greater than or equal to $0.2 \mathrm{mv}^{(2,14)}$. Subendocardial ischemia classically results in ST segment depression and $T$ wave inversion $^{(2,15)}$. In contrast our study showed that although ST segment depression was the more frequent form of ECG changes in NSTEACS, there was no significant difference in troponin positivity in them, nevertheless $T$ wave inversion was significantly associated with troponin positivity in male patients. Some studies have cast doubt on the prognostic value of isolated $T$ wave inversion ${ }^{(10,16)}$. These differences can be explained by the site of the lesion, as the proximal left anterior descending artery lesions usually produce deep $T$ wave inversion in chest leads ${ }^{(5)}$.

Non-specific or normal ECG was found in $24 \%$ of patients, equally distributed in male and female. $27 \%$ of them were troponin positive. Troponin positivity in patients with normal or non-specific ECG was significantly associated with male gender. According to randomized trial, males having chest pain with normal or non-diagnostic ECG are at higher risk for having ACS than women $^{(3,17)}$. In several studies, around $5 \%$ of patients with normal ECG who were discharged from the emergency department were ultimately found to have either an acute $\mathrm{Ml}$ or unstable angina ${ }^{(10,18,19)}$. It should be appreciated that a completely normal ECG in patients presented with suspicious symptoms does not exclude the possibility of ACS. 
New BBB block was found in $9 \%$ of patients with ACS and half of them were troponin positive. All of those with troponin positive BBB were males. It is known that transient episodes of BBB can occasionally occur during ischemic attack ${ }^{(10)}$.

A number of risk scores have been developed to predict short and midterm outcomes in patients with $\operatorname{ACS}^{(12,20,21)}$. Several variables have been associated with higher ischemic risk in different scoring systems ${ }^{(12)}$. Old age, heart failure, ST segment deviation and elevated cardiac markers were selected as independent risk factors by most predictive models ${ }^{(12)}$. The most popular risk scoring systems in clinical practice have been the TIMI risk score $^{(12,22)}$, the GRACE risk scores ${ }^{(12,20,23)}$, and to a lesser extent, the OURSUIT risk score ${ }^{(12,24)}$.

In our study, the appearance of specific ECG changes in patients with ACS was statistically associated with male sex, DM, hypertension, old age and previous history of vascular disease, $(p$ value $\leq 0.03$ ). Although the presence of current smoking was more common in patients who show specific ECG changes, but it was statistically not significant (Table 3). Age and male sex are associated with more severe coronary artery disease and consequently with an increased risk of unfavorable outcome ${ }^{(10)}$. Previous manifestations of coronary artery disease are also associated with more frequent subsequent events, also DM and hypertension ${ }^{(10)}$. Indeed most of the well-known risk factors for $C A D$ are also risk indicators for a worse prognosis in unstable coronary artery disease $^{(10,25)}$.

Positive troponin test was significantly more detected in male ( $p$ value 0.003 ). Association of specific ECG changes and positive troponin was stronger in male $(p=0.009)$ and smoker patients $(p$ value $=0.04$ )

These findings were comparable to a study of 1576 patients ${ }^{(17)}$, including 132 with ACS. Patients with ACS were older, more likely to be a man, hypertensive and an ex-smoker. The presence of DM was traditionally known to cause silent ischemia $^{(2,7)}$, which explains the lesser effects as a risk factor in our study because the selection of patients depends on the presence of typical anginal chest pain.

Troponin positivity, and appearance of specific ECG changes were significantly affected by the number of risk factors that victim harbors $(p$ value $=0.002$ ), all patients with troponin positive, and most patients with significant ECG changes (75\%) had two or more risk factors.

\section{CONCLUSION}

The most common ECG changes in patients with NSTE-ACS was ST segment depression, followed by $T$ wave inversion. About one fourth of patients with clinical diagnosis of NSTE-ACS, had a normal or non-specific ECG changes. Two third of patients had a negative troponin test. In our study NSTEMI (positive troponin test) was more frequently associated with $\mathrm{T}$ wave inversion, while in previous studies $^{(10,14,16)}$, ST depression was the more frequent ECG pattern in NSTEMI. Specific ECG changes were associated with male gender, diabetes mellitus, hypertension, and old age. Male gender was associated with specific ECG changes and troponin positivity. The number of risk factors had an important effect on ECG changes and troponin results.

\section{REFERENCES}

1. Grech E D, Ramsdale DR. Acute coronary syndrome: unstable angina and non ST segment elevation myocardial infarction. BMJ 2003; 326:1259-61.

2. Suraj A, Suriti K, William A. Diagnosis of acute coronary syndrome. Am Fam Physician. 2005;72(1): 119-126.

3. Weber M, Hamm C. Redefinition of myocardial infarction, relevance of biomarkers. Herz. 2008; 33(2): 115-21.

4. Torres M, Moayedi S. Evaluation of the acutely dyspniac elderly patient. Clin Geriat Med. 2007; 23(2): 307-25.

5. Newby DE, Crubb NR, Bradbury A. Cardiovascular disease: acute coronary syndrome: Davidson principles and practice of medicine $21^{\text {st }}$ edition. Elsevier. 2010: 588-89.

6. Scirice BN, Morrow BA. Troponins in acute coronary syndrome. Sein Vasc Med. 2003; 3: 363-74.

7. Braunwald E, Antman EM, Beaslley JW, et al. Acc/ AHA2002 guideline update for the management of patients with unstable angina and non ST segment elevation myocardial infarction- summery article. J Am Coll Cardiol. 2002; 40: 1366-74.

8. Alpert J S, Thygesen K, Antman E, Bassard JP. Myocardial infarction redefined - a consensus document of the Joint European Society of Cardiology/ American College of Cardiology Committee for the redefinition of myocardial infarction. J Am Coll Cardiol. 2000; 36: 959-69.

9. Chun AA, Mc Gee SR. Bedside diagnosis of coronary artery disease: a systemic review. Am J Med. 2004; 117(5): 334-43.

10. Bertrand ME, Chair ML, Simoon K, et al. Management of acute coronary syndromes: acute 
coronary syndromes without persistent ST segment elevation. European Heart J. 2000; 21:1406-32.

11. Fisch $\mathrm{C}$. Evolution of the clinical electrocardiogram. J Am Coll Cardiol.1989; 14(5):1127-1138.

12. Hector B, Francisco $F$. Use of risk scores in acute coronary syndromes. Heart. 2012; 98: 162- 68.

13. Graves EJ, Kozak LJ. Detailed diagnosis and procedures. Vital Health Statistics. 1998; 138:151.

14. Lioyd J, Camargo CA, Lapuerta P, Gingliono RP. Electrocardiographic and clinical predictors of acute myocardial infarction in patients with unstable angina pectoris. Am J Cardiol. 1998; 81: 1182- 86.

15. Goldberger AL. Clinical electrocardiography: a simplified approach $6^{\text {th }}$ ed. ST. Louis: Mosby. 1999: 81 100.

16. Miltenburg- van AJ, Simoons ML, Veerhook RJ, Bossuyt PM. Incidence and follow up of Braunwald sub groups in unstable angina pectoris. J Am Cardiol. 1995; 25: 1286-92.

17. Goodacre S, Pett P, Arnold J, et al. Clinical diagnosis of acute coronary syndrome in patients with chest pain a normal or non-diagnostic ECG. Emerg Med J. 2009; 26(12): 866- 70.

18. Mc Carthy B, Wong J, Selker H. Detecting acute ischemia in the emergency department. J Gen Int Med. 1990; 5: 365-73.

19. Pozen M, Agostino $R$, Selker $H$, A predictive instrument to improve $\mathrm{CCU}$ admission practice in acute ischemic heart disease: a progressive multicenter clinical trial. N Engl J Med. 1984;310: 1279-92.

20. Granger CB, Goldberg RJ, Dabbons O, et al. predictors of hospital mortality in the global registry of acute coronary events. Arch Intern Med. 2003; 163: 2345-53.

21. Mehran R, Pocock SJ, Nikolisky E, et al. a risk score to predict bleeding in patients with acute coronary syndrome. J Am Cardiol. 2010; 55: 2556-66.

22. Antman EM, Cohen M, Bernink PJ, et al. the TIMI risk score for unstable angina/ non ST elevation MI: a method for prognostication and therapeutic decision making. JAMA. 2000; 284: 835-42.

23. Fox KAA, Dabbous $\mathrm{OH}$, Gold berg RJ, et al. predictors of risk of death and the myocardial infarction in the six months after presentation of acute coronary syndrome: prospective multinational observational study (GRACE). BMJ. 2006; 333: 1091

24. Boersma E, Pieper KS, Steyerberg EW, et al. predictors of outcome in patients with acute coronary syndromes without persistent ST segment elevation: results of an international trial of 9461 patients, the PURSUIT Investigators. Circulation 2000; 101: 2557-67. 25. Campbell R, Wallentin L, Verheng F, Turpic A. Management strategies for a better outcome in unstable coronary artery disease. Clin Cardiol. 1998; 21: 314-22. 